\title{
Association between changes in coronary artery circulation and cardiac venous retention: a lesson from cardiac computed tomography
}

\author{
Rafal Mlynarski • Agnieszka Mlynarska • \\ Maciej Sosnowski
}

Received: 15 July 2012/ Accepted: 8 October 2012/Published online: 18 October 2012

(c) The Author(s) 2012. This article is published with open access at Springerlink.com

\begin{abstract}
To use computed tomography (CT) image data to measure a potential association between the implantation of coronary artery bypass grafts $(\mathrm{CABG})$ and changes in the coronary venous system has not yet been examined. In 112 (aged 59.4 $\pm 9.0 ; 45 \mathrm{~F}$ ) patients (pts.), a 64-slice CT angiography was performed. Patients were divided into 2 groups: CABG (56 pts.) and control (56 pts.)—without changes in coronaries. In each case, ten multi-planar reconstructions (MPR) and 3D volume rendering reconstructions using a $2 \mathrm{~mm}$ layer with ECG-gating, helical pitch: 12.8; rotation time: $0.4 \mathrm{~s}$ and average tube voltage: $135 \mathrm{kV}$ at $380 \mathrm{~mA}$. The visualization of the coronary veins was independently graded by 2 experts trained in CT. In the CABG group, the average number of visible coronary veins was $5.3 \pm 1.3$, while in the control group it was $3.1 \pm 1.1(p<0.001)$. Statistical differences were also observed for the following coronary veins: posterolateral (control $2.1 \pm 1.9$ vs. CABG $2.9 \pm 1.9 ; p<0.05$ ), lateral (control $2.2 \pm 1.7$ vs. CABG $3.1 \pm 1.3 ; p<0.01$ ) and anterolateral (control $0.5 \pm 0.9$ vs. CABG $1.3 \pm 1.0 ; p<0.001)$. Implantation of CABG influences the coronary venous system. In patients after CABG, the number of identifiable coronary veins is significantly higher as compared to that in subjects without
\end{abstract}

R. Mlynarski ( $₫) \cdot$ A. Mlynarska

Department of Electrocardiology,

Upper-Silesian Medical Centre, ul Ziolowa 45/47,

40-635 Katowice, Poland

e-mail: joker@mp.pl

R. Mlynarski · A. Mlynarska - M. Sosnowski

Unit of Noninvasive Cardiovascular Diagnostics, Upper-Silesian

Medical Centre, Katowice, Poland

M. Sosnowski

3rd Division of Cardiology, Medical University of Silesia,

Katowice, Poland changes in coronaries. This might suggest an association between changes in coronary artery circulation and cardiac venous retention.

Keywords CABG - Coronary veins ·

Computed tomography $\cdot$ Coronary circulation

\section{Introduction}

Knowledge of the importance of the coronary venous system has increased during recent decades mainly due to advances in electrophysiological procedures such as ablation or cardiac resynchronization [1-3]. In the past there was also an attempt to use the coronary venous system for the treatment of coronary artery disease (CAD); however, this method was not clinically appreciated [4].

It was shown that in some diseases like heart failure, $\mathrm{CAD}$ or myocardial infarction, the anatomy of the coronary veins was affected - this can be caused by changes in the flow. Information about changes in the venous anatomy is very important especially from an electrophysiologist's point of view because many patients who qualify for resynchronization suffer from the diseases mentioned above.

We hypothesized that advanced $\mathrm{CAD}$, in which the implantation of coronary bypass grafts (CABG) is necessary, can influence the coronary venous circulation and influence the venous anatomy. New imaging methods can be useful in finding an explanation for this phenomenon [5]. There have been several studies which have confirmed the usefulness of MSCT for the visualization of the coronary venous system, but none of them included patients with a previous bypass grafts implantation $[6,7]$. 
The purpose of this study was to evaluate the cardiac venous anatomy in patients after coronary artery bypass graft surgery.

\section{Methods}

112 patients (aged $59.4 \pm 9.0 ; 45$ females) in 2 equal groups were included into the trial:

- 56 patients with previously implanted bypass grafts (CABG group) in which MSCT was performed to check the patency of the grafts.

- 56 patients included due to a suspicion of CAD without changes within the coronaries (controls).

Patients were excluded if stenosis in the coronaries was present (controls), if they had atrial fibrillation, frequent premature heartbeats, renal insufficiency (serum creatinine $>1.2 \mathrm{mg} / \mathrm{dl}$ ), hyperthyreosis, any known allergy to nonionic contrast agents or a previously implanted pacemaker with unipolar leads [9].

A 64-detector row cardiac computed tomography (CT) was performed on each patient. In all of the patients, the coronary venous system was analyzed including the number of visible coronary veins and the type of veins visualized (coronary sinus, anterior, antero-lateral, lateral, postero-lateral, posterior veins and middle cardiac veins). The nomenclature of those vessels was based on earlier published methodology [8].

Computed tomography was performed using an Aquilion 64 scanner (Toshiba Medical Systems, Japan). Scanning with retrospective ECG-gating was performed during a breath-hold using 64 slices with a collimated slice thickness of $0.5 \mathrm{~mm}$. A breath-hold examination was performed in order to adjust the scanner settings.

The helical pitch was 12.8 (best mode) and the rotation time was $0.4 \mathrm{~s}$. The tube voltage was strictly dependent on the patient's body mass index (BMI): for BMI $<23.9$, it was $120 \mathrm{kV}$ at $330 \mathrm{~mA}$, for $\mathrm{BMI}=24.0-29.9$, it was $135 \mathrm{kV}$ at $380 \mathrm{~mA}$ and for $\mathrm{BMI}>30.0$, it was $135 \mathrm{kV}$ at $430 \mathrm{~mA}$. We used a pre-selected region of interest (ROI) in the descending aorta. Triggering started at 180 Hounsfield units. On average, $100 \mathrm{ml}$ of non-ionic contrast agent (Ioperamid, Ultravist 370, Schering, Germany) was administered to each patient during the examination at an average rate of $4.5 \mathrm{ml} / \mathrm{s}$ in the control group and $130 \mathrm{ml}$ at an average rate $4.5-05 \mathrm{ml} / \mathrm{s}$ in the CABG group in three phases.

The cut-off for heart rate (HR) was set at $65 \mathrm{bpm}$. If the HR was higher, metoprolol succinate (Betaloc, Astra Zeneca, Sweden) at a dosage of 5-10 mg was administered intravenously, unless contraindicated. If the expected HR slowing did not occur, the patient was excluded from the study. Sublingual nitroglycerin was not administered before image acquisition.

Reconstructions of data were performed on Vitrea 2 workstations (Vital Images, USA; software version 3.9.0.0 and 5.1). In each case, ten (10) axial image series and 3D volume rendering reconstructions were created from 0 to $90 \%$ R-R intervals (step $10 \%$ ) using a $2.0 \mathrm{~mm}$ slice thickness to reduce the large amount of data.

Optimal phases were chosen based on the quality of the visualization using a scale that was developed and published by our team earlier (Table 1) [10].

Vessels were graded by 2 experts trained in MSCT and experienced in the visualization of the coronary venous system (more than 250 venograms evaluated in MSCT). Reproducibility of the phase determination was evaluated using the Bland-Altman method and by the calculation of the inter-rater agreement coefficient kappa. Correlations between data were calculated by means of the Spearman rank coefficient (MedCalc Soft, Belgium).

The local Ethics Committee approved the study protocol. The study protocol complied with the version of the Helsinki Convention that was current at the time the study was designed.

\section{Results}

The characteristics of the patients included are presented in Table 2.

The number of visible cardiac veins was highly reproducible (inter-rater agreement kappa: 1.000; standard error: 0.000), while intra-observer agreement was excellent (mean difference 0, inter-rater agreement kappa 1.0).

Table 1 The scale used for the visualization of arteries and veins [10]

\begin{tabular}{ll}
\hline Score & Description \\
\hline 0 & No vessel(s) present ${ }^{\mathrm{a}}$ \\
$1^{\mathrm{b}}$ & $\begin{array}{l}\text { Vessel, length less than } 5 \mathrm{~mm} \text {, weakly contrasted and/or with } \\
\text { a number of artifacts }\end{array}$ \\
2 & Between score 1 and score 3 \\
3 & Vessel more than $1 \mathrm{~cm}$ long, better contrasted. Sometimes \\
& areas not visible or artifacts occurred. \\
4 & Between score 3 and score 5 \\
5 & Vessel well contrasted, clearly visible on the entire length of \\
& the vessel
\end{tabular}

\footnotetext{
${ }^{a}$ When a vein is not visualized in any of the phases, it has a grade of 0 . However, this does not necessarily mean that the vein is absent-it could be e.g. very small and therefore lower than the resolution of the CT scanner

${ }^{\mathrm{b}}$ Grade 1 means that a vessel is present but very poorly visualized
} 
Similarly, there was a very good agreement between observers in the evaluation of the quality of the reconstructions $(95 \% \mathrm{CI}-0.945$ to 1.000 , inter-rater agreement kappa 0.974). There were similar results in the repeated evaluation of the score by the same observer $(95 \%$ CI 0.959-1.0000 and kappa 0.983).

A good quality visualization was obtained for both groups; however, the average quality of the visualization was statistically better in the CABG group $(4.3 \pm$ 0.8 points) as compared to the control group $(3.9 \pm 1.1$, $p<0.01)$.

The average number of coronary veins was $5.3 \pm 1.3$ in the CABG group, while in the control group, it reached $3.1 \pm 1.1(p<0.001)$. Statistical differences were also observed for the following coronary veins: posterolateral, lateral and anterolateral. No statistical differences were observed for the radical veins: posterior vein and anterior vein. Relations in the number of coronary veins analyzed are presented in Table 3. An example of the anatomy from both groups is presented in Fig. 1. Additionally, the distribution of the occurrence of veins analyzed is presented in Fig. 2.

Table 2 Characteristics of the patients included

\begin{tabular}{llll}
\hline & CABG & Control & $p$ \\
\hline Hemodynamic parameters $^{\mathrm{a}}$ & & & \\
Ejection fraction (\%) & $58.1 \pm 13.6$ & $63.9 \pm 7.9$ & 0.0182 \\
EDV (ml) & $156.4 \pm 41.6$ & $135.5 \pm 42.8$ & 0.0375 \\
ESV (ml) & $71.7 \pm 43.1$ & $49.5 \pm 21.9$ & 0.003 \\
Stroke volume (ml) & $87.2 \pm 20.3$ & $83.2 \pm 19.1$ & NS \\
Cardiac output (l/min) & $5.4 \pm 1.4$ & $5.3 \pm 1.9$ & NS \\
Myocardial mass (g) & $150.6 \pm 29.5$ & $125.8 \pm 41.4$ & 0.0029 \\
Myocardial volume (ml) & $143.1 \pm 28.3$ & $113.4 \pm 36.1$ & 0.0003 \\
Risk factors & & & \\
Heart rate (bpm) & $62.2 \pm 8.6$ & $62.3 \pm 15.5$ & NS \\
Hypercholesterolemia (\%) & 100 & 26.8 & 0.0001 \\
Hypertension (\%) & 64.3 & 14.3 & 0.0003 \\
Diabetes (\%) & 50 & 8.9 & 0.0004 \\
Smoking (\%) & 35.7 & 21.4 & NS \\
\hline
\end{tabular}

${ }^{a}$ Values by cardiac computer tomography

Table 3 Average number of coronary veins visualized in both groups

\begin{tabular}{llll}
\hline & \multicolumn{2}{l}{ Average $\pm \mathrm{SD}$} & $p$ \\
\cline { 2 - 3 } & CABG & Control & \\
\hline Posterior vein & $1.59 \pm 1.86$ & $1.30 \pm 1.79$ & $\mathrm{NS}$ \\
Posterolateral vein & $2.89 \pm 1.86$ & $2.05 \pm 1.91$ & 0.022 \\
Lateral vein & $3.14 \pm 1.28$ & $2.17 \pm 1.65$ & 0.019 \\
Anterolateral vein & $1.32 \pm 0.97$ & $0.51 \pm 0.93$ & 0.000 \\
Anterior vein & $2.28 \pm 1.37$ & $1.93 \pm 1.47$ & $\mathrm{NS}$ \\
\hline
\end{tabular}

We also observed that in older subjects the number of veins increased in both groups. A statistically significant correlation $(\mathrm{r}=0.245 ; p<0.05)$ was found only for control group. Such a correlation was not observed in the CABG group. Both correlations are presented graphically in Fig. 3.

\section{Discussion}

To the best of our knowledge, there has been no research to date that shows the influence of cardiovascular pathologies on the anatomy of coronary veins. Because the role of the coronary venous system is very important as a "natural safety valve" in many diseases, the influence of any modification of coronary circulation into the coronary venous system has been the subject of some research and scientific hypotheses. More than 100 years ago, Pratt et al. presented the hypothesis of the perfusion of isolated feline hearts by the coronary venous system $[4,11]$. Since that time, this hypothesis has been explored by Beck-anastomosis to arterial vessels and Lillehei et al. [12, 13]—using retroperfusion during cardiac surgery. This concept has not been fully utilized clinically because arterial surgical revascularization methods have been shown to be both safe and efficacious. On the other hand, many patients suffer from additional diseases such as heart failure or heart rhythm disorders where cardiac resynchronization (CRT) implantation or ablation is indicated. In such cases, cannulation of the coronary sinus or (in CRT) access to the coronary vein has to be performed. Our earlier studies regarding the visualization of the coronary venous system in MSCT documented the anatomy of coronary veins in subjects without changes in coronary arteries [8, 14]. Similar research has also been performed by other researchers $[6,15,16]$. During our clinical practice, we observed that in most patients qualified for CT coronary angiography for the evaluation of the patency of bypass grafts, the coronary venous system is more developed as compared to subjects without CAD. This observation contradicted information from electrophysiologists who found that patients have many problems after CABG when a left ventricle lead is implanted. Our results are in full agreement with the research of Hajaghaei et al. [17]. The authors showed a significant increase in the diameter of the coronary sinus in patients after CABG. They also confirmed that the coronary flow reserve (CFR) was significantly higher after cardiac surgery.

The results of this study might be explained by the fact that the implantation of CABG changes the distribution of pressures within the arterial and venous vessels. Higher pressure in the coronary venous system can cause the expansion of veins as a compensation mechanism. Also, 
Fig. 1 Example of the 3D anatomy of the heart in the $C A B G$ and control groups. Lateral view of the heart, 3D volume rendering reconstruction

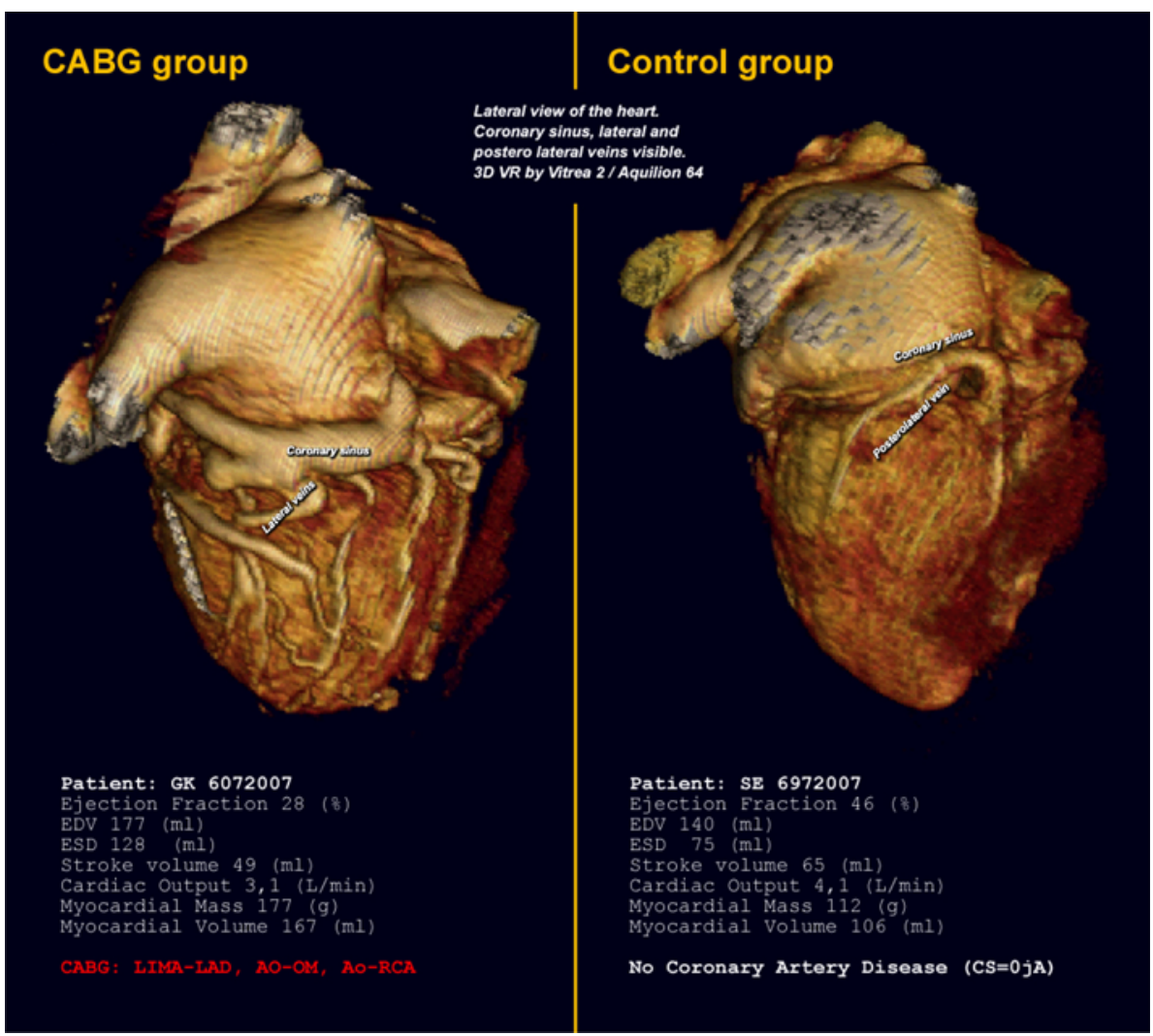

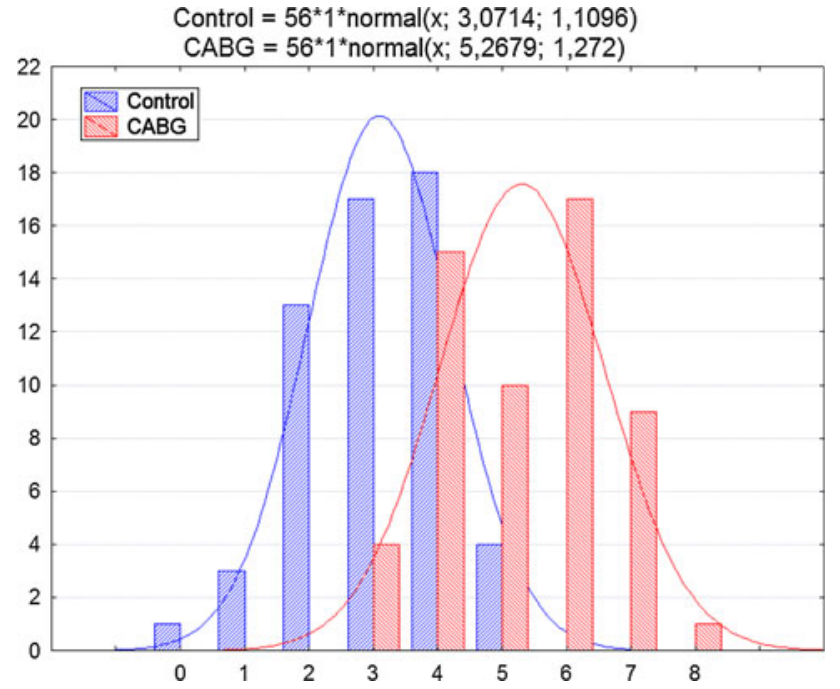

Fig. 2 Dispositions of the occurrences of veins analyzed in the groups studied

one might hypothesize that increased coronary venous pressure can support the ischemic myocardium in the presence of advanced atherosclerotic lesions. Changes in contrast enhancement, as first proposed by Rybicki et al. [18], and then expanded upon by Steigner et al. [19] followed by Chow et al. [20] have been shown to contain additional diagnostic information. Our manuscript provides additional data to support the concept that coronary enhancement is related to flow, and more specifically that alterations in coronary blood flow patterns can be measured, at least in part, by CT. However, anatomo-pathology studies are necessary to confirm these explanations.

\section{Limitations of the study}

Presented study is observational study only-we did not perform two examinations on each patient (before and after cardio surgery) due to the large dose of radiation. We are aware that the main aim of an MSCT examination in the included patients was the evaluation of the coronary arteries. Thus, as no special scan and contrast protocol for venous enhancement was used, in certain cases the existing veins could not be visualized. However, as the same protocol (arterial phase) was used in both groups, the observed differences in the venous anatomy are reliable. Our results do not explain why difficulties during implantation of CRT in patients after CABG occurred; we only documented that for some reason the number of coronary veins increases after bypasses. There is no other research describing the venous anatomy in patients after CABG; therefore we do not have the possibility to discuss these results with other authors. 

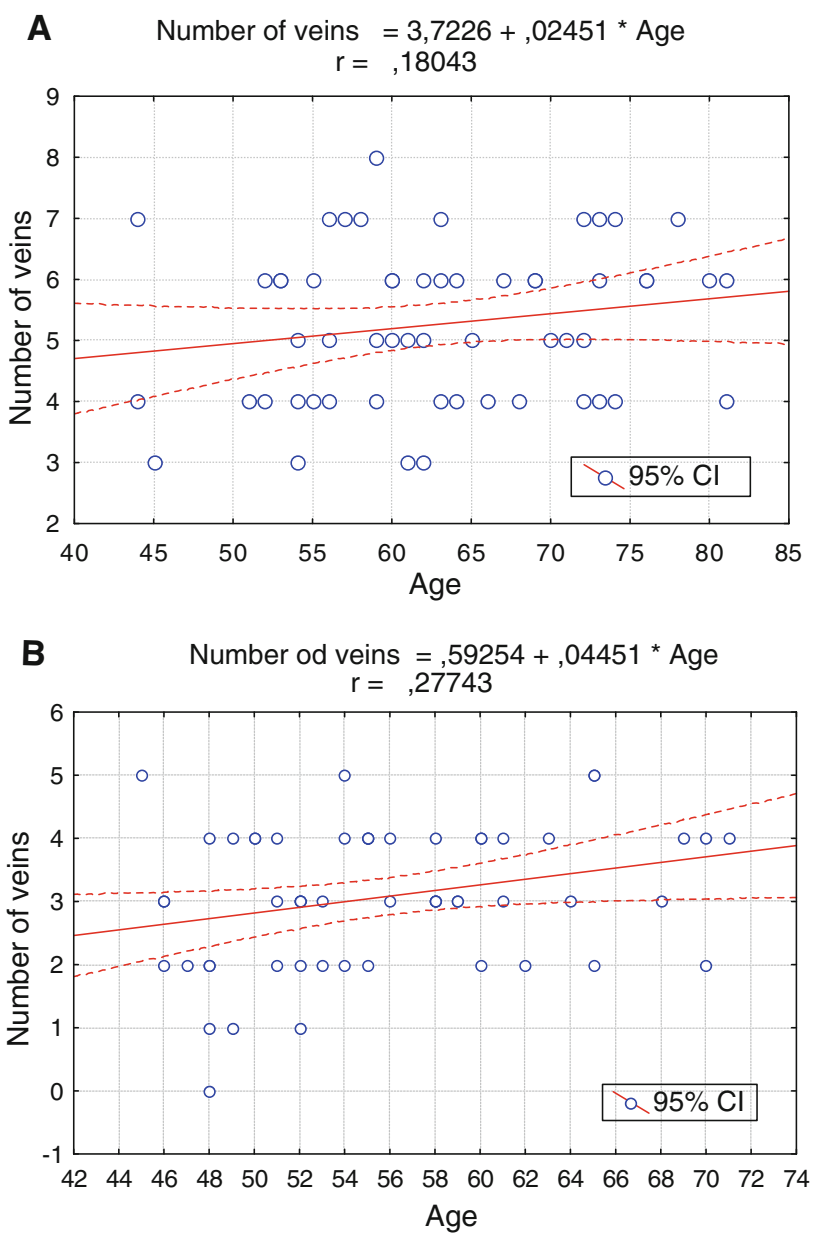

Fig. 3 Correlations between age and the number of visible veins. a CABG group; b control group

\section{Conclusions}

Advanced CAD where implantation of CABG is needed has a significant influence on the coronary venous system. The number of identifiable coronary veins is significantly higher in patients after CABG as compared to subjects without changes in coronaries. This might suggest an association between changes in coronary artery circulation and cardiac venous retention. The clinical significance and pathophysiological meaning of this finding requires further studies.

Acknowledgments The authors are indebted to the radiology technologists for their technical support.

\section{Conflict of interest None.}

Open Access This article is distributed under the terms of the Creative Commons Attribution License which permits any use, distribution, and reproduction in any medium, provided the original author(s) and the source are credited.

\section{References}

1. Giraldi F, Cattadori G, Roberto M, Carbucicchio C, Pepi M, Ballerini G, Alamanni F, Della Bella P, Pontone G, Andreini D, Tondo C, Agostoni PG (2011) Long-term effectiveness of cardiac resynchronization therapy in heart failure patients with unfavorable cardiac veins anatomy comparison of surgical versus hemodynamic procedure. J Am Coll Cardiol 58:483-490

2. Ypenburg C, van der Wall EE, Schalij MJ, Bax JJ (2008) Imaging in cardiac resynchronisation therapy. Neth Heart J 16(Suppl 1): S36-S40

3. Miyamoto K, Tsuchiya T, Yamaguchi T, Nagamoto Y, Ando S, Sadamatsu K, Tanioka Y, Takahashi N (2011) A new method of a pulmonary vein map to identify a conduction gap on the pulmonary vein antrum ablation line. Circ J 75:2363-2371

4. Kassab GS, Navia JA, March K, Choy JS (2008) Coronary venous retroperfusion: an old concept, a new approach. J Appl Physiol 104:1266-1272

5. Manzke R, Binner L, Bornstedt A, Merkle N, Lutz A, Gradinger R, Rasche V (2011) Assessment of the coronary venous system in heart failure patients by blood pool agent enhanced whole-heart MRI. Eur Radiol 21:799-806

6. Doganay S, Karaman A, Gündogdu F, Duran C, Yalcin A, Kantarci M (2011) Usefulness of multidetector computed tomography coronary venous angiography examination before cardiac resynchronization therapy. Jpn J Radiol. 29:342-347

7. Mlynarski R, Sosnowski M, Wlodyka A, Kargul W, Tendera M (2009) A user-friendly method of cardiac venous system visualization in 64-slice computed tomography. Pacing Clin Electrophysiol 32:323-329

8. Mlynarski R, Mlynarska A, Sosnowski M (2011) Anatomical variants of coronary venous system on cardiac computed tomography. Circ J 75:613-618

9. Mlynarski R, Sosnowski M, Mlynarska A, Tendera M (2012) Computed tomography in patients with cardiac pacemakers: difficulties and solutions. Heart Vessels. [electronic version]

10. Mlynarska A, Młynarski R, Kargul W, Sosnowski M (2011) Quality of visualization of coronary venous system in 64-slice computed tomography. Cardiol J. 18:146-150

11. Pratt FH (1897) The circulation through the veins of Thebesius. J Boston Soc Med Sci J. 1:29-34

12. Beck CS (1949) Revascularization of the heart. Surgery $26: 82-88$

13. Lillehei CW, Dewall RA, Gott VL, Varco RL (1956) The direct vision correction of calcific aortic stenosis by means of a pumpoxygenator and retrograde coronary sinus perfusion. Dis Chest 30:123-132

14. Mlynarski R, Mlynarska A, Tendera M, Sosnowski M (2011) Coronary sinus ostium: the key structure in the heart's anatomy from the electrophysiologist's point of view. Heart Vessels 26:449-456

15. Van de Veire NR, Marsan NA, Schuijf JD, Bleeker GB, Wijffels MC, van Erven L, Holman ER, De Sutter J, van der Wall EE, Schalij MJ, Bax JJ (2008) Noninvasive imaging of cardiac venous anatomy with 64-slice multi-slice computed tomography and noninvasive assessment of left ventricular dyssynchrony by 3-dimensional tissue synchronization imaging in patients with heart failure scheduled for cardiac resynchronization therapy. Am J Cardiol 101:1023-1029

16. Van de Veire NR, Schuijf JD, De Sutter J, Devos D, Bleeker GB, de Roos A, van der Wall EE, Schalij MJ, Bax JJ (2006) Noninvasive visualization of the cardiac venous system in coronary artery disease patients using 64-slice computed tomography. J Am Coll Cardiol 48:1832-1838

17. Hajaghaei M, Maleki M, Salehi HR, Ojaghi Z, Noohi F (2007) Coronary flow reserve measurement in the coronary sinus in pre and post CABG status. Iranian cardiovasc. res. J. 1:87-91 
18. Rybicki FJ, Otero HJ, Steigner ML, Vorobiof G, Nallamshetty L, Mitsouras D, Ersoy H, Mather RT, Judy PF, Cai T, Coyner K, Schultz K, Whitmore AG, Di Carli MF (2008) Initial evaluation of coronary images from 320-detector row computed tomography. Int J Cardiovasc Imaging 24:535-546

19. Steigner ML, Otero HJ, Cai T, Mitsouras D, Nallamshetty L, Whitmore AG, Ersoy H, Levit NA, Di Carli MF, Rybicki FJ
(2009) Narrowing the phase window width in prospectively ECG-gated single heart beat 320-detector row coronary CT angiography. Int J Cardiovasc Imaging 25:85-90

20. Chow BJ, Kass M, Gagné O, Chen L, Yam Y, Dick A, Wells GA (2011) Can differences in corrected coronary opacification measured with computed tomography predict resting coronary artery flow? J Am Coll Cardiol 57:1280-1288 